\title{
INTEGRATING CHILDREN'S MENTAL HEALTH IN PRIMARY HEALTH CARE
}

\author{
HAROLD LIPTON, ELAINE RAIVIO, ELLEN PERRAULT, BARBARA \\ BRYDEN, VIRGINIA CAPUTY, LINDA BINDING, DEBORAH PACE, \\ ALLAN DONSKY, ALANDA PETERS, and KRISTY PLOTSKY \\ Healthy Minds/Healthy Children Outreach Service, Calgary Health Region \\ Calgary, Alberta \\ JESSICA AYALA \\ University of Calgary, Alberta \\ CAROLYN PADA \\ Mount Royal College, Calgary, Alberta
}

\begin{abstract}
The Healthy Minds/Healthy Children Outreach Service is part of Alberta's strategy to increase the capacity of primary care providers to identify and treat children and youth with mental health issues. Program development, implementation, and interim results are presented. Particular challenges connecting with Aboriginal communities, developing resources, and providing clinical consultation and online professional development are discussed. Evaluation data suggest that this form of collaborative, educational, interprofessional service is a promising approach to narrowing the care gap for children with mental health issues.
\end{abstract}

Reported prevalence rates for mental health diagnoses among Canadian children and youth are 15 to $20 \%$ (Adlaf, Paglia, \& Beitchman, 2002; Kirby \& Keon, 2006; Kutcher, 2007; Steele et al., 2004; Steinhauer, 1999), yet 75-85\% of young Canadians with mental health concerns do not receive any specialized mental health treatment (Kataoka, Zhang, \& Wells, 2002; McEwan, Waddell, \& Barker, 2007; Steinhauer, 1999). Although mental health services are often provided by family physicians (Kutcher, 2007; Steele et al., 2004), most consider themselves unprepared to serve this population (Steinhauer, 1999) or feel that they lack training (Cockburn \& Bernard, 2004; Curran \& Fleet, 2005) and skill in this area (Steele et al., 2003).

The integration of children's mental health in primary health care was supported by funding from the Primary Health Care Transition Fund through the Alberta Health and Wellness Capacity Building Fund and the Alberta Health and Wellness Mental Health Innovation Fund. The writing of this article was a collaborative effort reflective of the practice philosophy of the Healthy Minds/Healthy Children Outreach Service. 
The capacity of family physicians to treat children with mental illness is of particular concern as children can be affected more acutely and severely than adults when their mental health needs are not met; moreover, undiagnosed and untreated mental illness in childhood can have lifelong consequences (Kirby \& Keon, 2006). A significant proportion of adult mental illness is now thought to have clearly discernible roots in childhood and adolescence (Canadian Collaborative Mental Health Initiative, 2006; Health Canada, 2002).

The shortage of physicians in Canada (McElroy, 2004) has widened this care gap, particularly in rural areas. The even greater shortage of child and adolescent psychiatrists makes the provision of adequate mental health services for this population one of the biggest challenges facing rural communities (Collaborative Working Group on Shared Mental Health Care, 2000).

To begin to address these challenges, health care reform in Canada has called for more comprehensive primary care services, integration of specialized (such as mental health) services within primary care, improved continuing professional development for providers, and better access to services in remote communities (Kirby \& Keon, 2006; Premier's Advisory Council on Health for Alberta, 2001). All of these recommendations place greater emphasis on interprofessional collaboration at the primary care level in order to provide better patient care (Johnston, Smylie, \& Prince, 2004). An increasing number of shared or collaborative mental health care programs have developed in Canada, many targeted at populations that are traditionally underserved, including rural and remote communities (Pauzé, Gagné, \& Pautler, 2005). Typically, these initiatives involve mental health specialists such as psychiatrists, psychologists, social workers, or nurse therapists providing expertise to primary care physicians (PCPs) and/or their patients.

\section{HEALTHY MINDS/HEALTHY CHILDREN}

\section{Program Development}

The Southern Alberta Child and Youth Health Network (SACYHN), formed in 2001, is a collaborative network of parents and organizations serving children that includes government ministries and agencies, health authorities, university faculties (medicine, dentistry, and social work), and not-forprofit agencies. Such networks are an effective method of linking services and do not require the creation of new service delivery systems (Dolinski, 2005).

SACYHN's mission is to support the provision of high-quality health services to children and youth proximal to their communities; this mission includes the development of outreach services. As part of its framework, SACYHN focuses on improving the knowledge and practice of primary care providers to achieve positive health outcomes. In 2003, SACYHN received funding from the federal Primary Health Care Transition Fund (PHCTF) to build primary care capacity in early identification and intervention in children's mental health. This funding launched the Healthy Minds/Healthy Children (HM/HC) mental health project and supported it until 2006, when funding was obtained from the Alberta Health and Wellness Mental Health Innovation Fund.

The PHCTF funding of $\mathrm{HM} / \mathrm{HC}$ mandated that priority be given to building linkages with primary care physicians, particularly in rural parts of southern Alberta. In addition, the Treaty 7 agreement 
between governments and First Nations of southern Alberta communicates a clear intent for the improvement of health care for First Nations people. Mental health care for First Nations children and youth was, and continues to be, a priority for the province and for Healthy Minds/Healthy Children.

An intersectoral working group was formed that included representatives from health regions, SACYHN, Treaty 7 First Nations, University of Calgary's Faculty of Social Work and Faculty of Medicine, and the Calgary Health Region's Department of Family Medicine, Department of Child and Adolescent Psychiatry, and Department of Pediatrics. An initial needs assessment and literature review confirmed that primary care physicians felt that they lacked knowledge, skills, and confidence in the area of child and youth mental health, and identified the acquisition of information and the provision of clinical support as essential to assist physicians to recognize and manage the mental health concerns of children and adolescents. The working group guided the development of the program, maintaining a focus on the goal of building capacity at the primary care level.

Healthy Minds/Healthy Children aimed to facilitate the acquisition and dissemination of knowledge and skill in children's mental health by primary care physicians, to bring services closer to children and families, and to build coordination of those services across the health regions and First Nations of southern Alberta. HM/HC adopted a consultation-liaison model in which the primary care physician was present at all activities (Craven \& Bland, 2002); this focus on contact with physicians rather than on direct patient services allowed for a broader dissemination of knowledge and skill (Steinhauer, 1999). The use of technology provided an opportunity to span wide geographic areas and to offer rural and isolated practitioners the same access to consultation and education as their urban counterparts. Educational programming and resource tools were also utilized to achieve the program's goals.

\section{Services Provided}

Clinical consultations. Clinical consultation was offered to primary care physicians either at the point of care, via videoconferencing, or by telephone. Consultations were provided by a clinical specialist with an option to include a child/adolescent psychiatrist; some also involved the child, parents, caregivers, and other professionals, such as school personnel and social workers. Wherever possible, local linkages were facilitated or supported between physicians and nearby mental health services.

The HM/HC team ensured that appropriate standards governing the sharing of information were followed, such as obtaining consent when required. With respect to issues of liability and accountability of collaborating professionals (Canadian Medical Protective Association, 2005), the primary care physician retained clinical responsibility for ongoing care while the HM/HC team maintained awareness of scope of practice mandates established by regulatory bodies.

The HM/HC clinical consultants and psychiatrist met regularly to discuss the care of children with complex needs, generate additional recommendations for treatment and follow-up, and provide collegial support. The psychiatrist, as a physician, had a unique role at these rounds in addressing medical and medication issues. These clinical meetings were also opportunities to review and plan $\mathrm{HM} / \mathrm{HC}$ program strategies for continued provision of clinical supports to primary care physicians across the catchment area. 
Since 2003, nearly 600 consultations have been completed; $7 \%$ included the patient, and $1 \%$ of the consultations were with a patient alone. First Nations children comprised over one third of all consultation activity. Eight percent of consultations led immediately to a referral to more specialized care. Of the $63 \%$ of consultations for which a diagnosis was recorded, $60 \%$ involved disruptive behaviour, anxiety, or mood disorders. Overall, feedback from primary care physicians regarding the consultations was positive, particularly regarding the interprofessional aspect of the service. Clinicians from remote locations appreciated the opportunity to discuss clinical concerns with other professionals.

Education. The mental health education of primary care practitioners and physician residents has been recognized as critical to optimizing their role in providing care (Health Canada, 2002), although simple information dissemination does not, on its own, appear to result in clinical practice change (Davis et al., 1999; Kaner, Steven, Cassidy, \& Vardy, 2003). In 2005, HM/HC, in partnership with the Alberta Mental Health Board, Capital and Calgary Health Regions, SACYHN, and the University of Calgary's Faculty of Social Work, developed a Continuing Professional Development (CPD) program to aid in narrowing the care gap in mental health at the primary care level (Curran \& Fleet, 2005). Continuing professional education has been cited as an effective way of building the skills and knowledge required for competence in and conformity to standards of professional conduct (Harvey, 2004). It has also been recognized for its utility in reducing professional job distress (Kushnir, Cohen, \& Kitai, 2000) and in helping professionals cope with rapid changes in workforce, technology, and models of care provision (Towle, 2000).

E-learning was selected as the primary means of delivering the program because of its effectiveness for distance learning (Ayala, 2007). Online learning is popular, efficient, convenient, and accessible (Bassoppo-Moyo, 2006; Billings \& Rowles, 2001; Curran \& Fleet, 2005; Mamary \& Charles, 2000; Richardson \& Norris, 1997; Ryan \& Waterston, 2000). The Internet is increasingly popular as an information source for physicians (Curran \& Fleet, 2005) and effective in making information available to PCPs for their use in the provision of mental health care (Barnes, 2004; Peterson, 1999). As an alternative to the online format, curriculum material was also presented live or via videoconferencing for those without computer or Internet access.

Curriculum for the online program was selected from feedback received in the PCP needs assessment, from previous registrant input, from consultant contact with service providers, and from an annual online survey and ongoing learning platform that assesses learning needs through the project website. A selection of current and past topics is available on HM/HC's website. ${ }^{1}$ The heterogeneous needs of learners were accommodated by offering multiple levels of graduated complexity in many learning modules. The interprofessional $\mathrm{HM} / \mathrm{HC}$ advisory committee ensures that learning curricula remain current.

Experienced clinical practitioners in Alberta were invited to produce the HM/HC online CPD modules. The presentations consisted of PowerPoint slides with voice-over narration, and presenters were encouraged to give the material a practical focus for primary care physicians. Included with the presentations were related reading resources, handouts, lists of local treatment resources, text-based facilitated asynchronous discussion, and an online evaluation. The HM/HC clinical consultants also participated in the provision of the online CPD program, providing clinical educational comments in the online discussions, and assisting with the selection of resource materials and references for each module. 
Accreditation was received from the College of Family Physicians of Canada, the Royal College of Physicians and Surgeons, and the Alberta College of Social Workers, and registrants could accumulate professional credits for participation. The CPD was promoted to all family/general practitioners, pediatricians, psychiatrists, and mental health clinics in Alberta.

Growth of the program has been exponential, and by the fourth session of modules, over 400 practitioners had participated in a total of 41 modules. Of participating professionals, $40 \%$ were rural practitioners (serving communities of less than 19,000 people). Registrants came from the fields of family medicine, psychiatry, pediatrics, psychology, social work, nursing, and other related mental health professions. Although fairly high dropout or non-completion rates have been reported for distance education and Internet-based courses (Chyung, 2001; Morgan \& Tam, 1999; Saba, 2000), in this program, an average of $79 \%$ of registrants participated in the discussion section of the online modules; of those, $76 \%$ completed module evaluations.

In the module evaluation, registrants were asked to rate a number of items on a 5-point Likert scale $(1$ = strongly disagree, 5 = strongly agree $)$; the modules have consistently been rated highly. Results from the 17 modules in the third session are presented in Figure 1. Respondents strongly agreed that the modules were relevant and of high quality, and that participation increased their knowledge and was likely to improve their skills and patient outcomes.

\section{Figure 1}

Online CPD (Session 3) Participant Feedback $(N=206)$

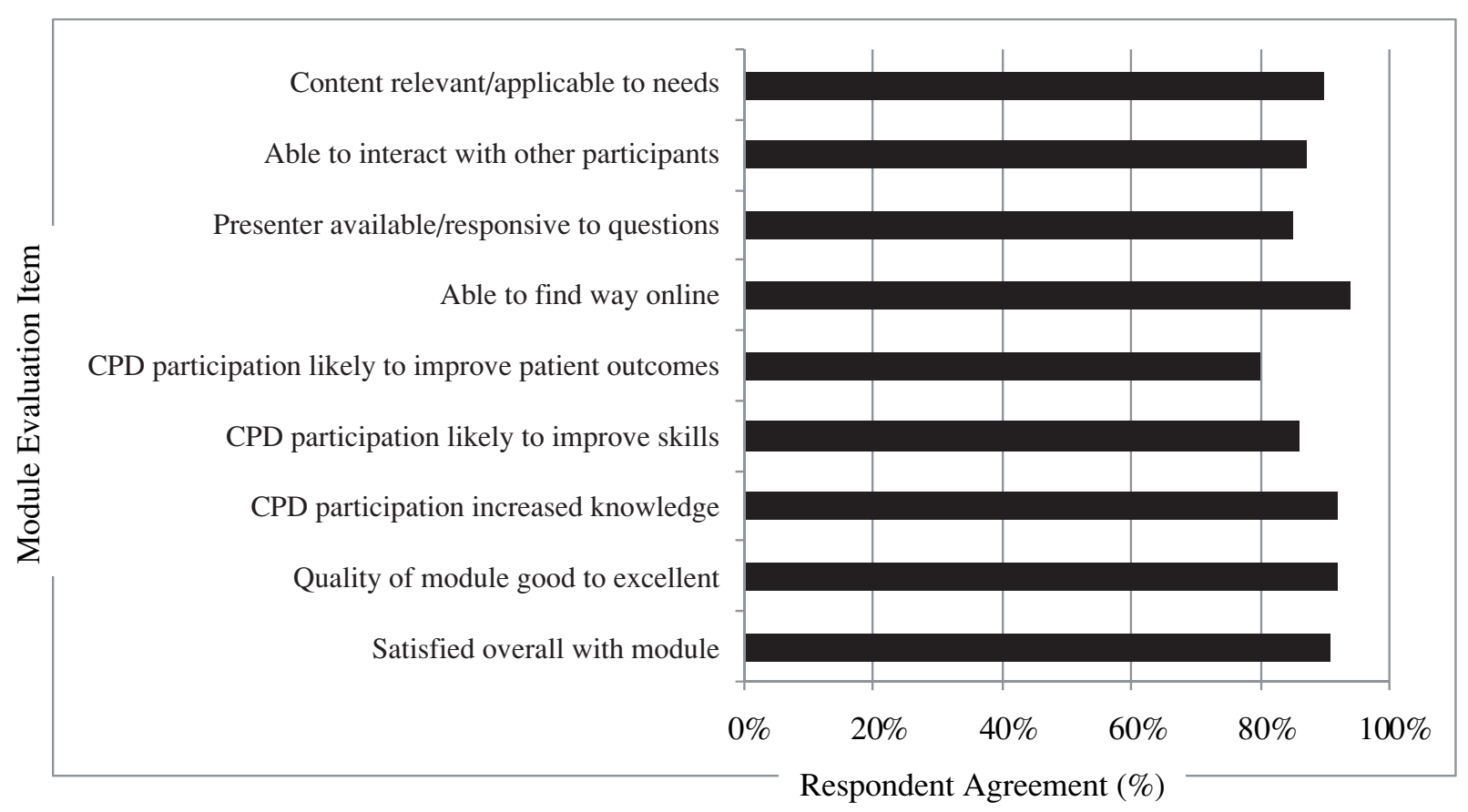

Note $. \mathrm{CPD}=$ Continuing Professional Development. 
Interactivity can significantly enhance continuing education (Davis et al., 1999), and some registrants reported that the interprofessional and interactive nature of the program helped them to value each other's work and unique contribution to children's mental health care and increased their willingness to work together more. Registrants reported that they found the discussions-with presenters and with each other-most useful to their learning and articulated the benefits of learning across professions, provided that the source of information was perceived as credible. Participants also commented positively on the practical focus of the discussions and on the convenience of the online medium. One of the most frequent suggestions for improvement was a request for additional practical tools and applications.

Resource tools. HM/HC also developed and distributed a number of resource tools. Examples include information prescriptions, which are itemized lists of books, videos, brochures, and websites that provide general information for parents and professionals on selected topics. The material is reviewed to ensure that it is current, evidence-based, and at an appropriate literacy level for its intended audience. The prescriptions were produced in collaboration with SACYHN's Family and Community Resource Centre. HM/HC was the lead developer for five prescriptions dealing with anxiety, depression, challenging behaviour, substance abuse, and bullying; additional topics are awaiting printing.

Another resource is a desk reference to aid the primary care physician in identifying and managing common mental health concerns and in distinguishing which patients should be referred to specialized or tertiary care. Diagnostic screens, questionnaires, treatment planners, and background information are offered in this reference, which is available in electronic and paper formats. The content, as with the information prescriptions, was driven by feedback from physicians. To date, more than 1,600 copies of the desk reference have been circulated, primarily in electronic form, to primary care physicians, other health care professionals, and children's services such as school-based services. In response to feedback, a second edition was developed that incorporated a more user-friendly format and additional chapters. Anecdotal feedback accompanying requests for additional copies stated that these manuals were a useful aid to daily practice.

In addition, Healthy Minds/Healthy Children created a website to make its resources available, promote continuing professional development, and provide links and suggestions for parents and practitioners to other related resources. Visits to the website have increased significantly since the baseline access rate was established in the fall of 2007; the online professional development and family resource pages have been particularly popular.

\section{Lessons Learned}

Initial uptake of outreach services was slow, possibly because the delivery model required a shift to collaborative activity that was difficult for physicians to fit into their busy schedules. They were also accustomed to being compensated on a fee-for-service basis and were reluctant to participate in collaborative care when much of the work was not remunerable under existing provincial arrangements. Alternative payment plans have since been developed in Alberta that can accommodate innovative methods of delivering care (Hasselback, Halma, Wedel, \& Janz, 2003). 
In some health regions, there were questions regarding how the offered service fit with local needs and priorities. Although regional and local management support was helpful in establishing linkages, the strongest connections were made where front-line primary care physicians enthusiastically embraced the collaborative capacity-building model. Consultation linkages often progressed from an intermittent phone contact between the primary care provider and HM/HC consultant when a particularly challenging situation occurred to more organized team consultations. These group activities consisted of a rich blending of multiple perspectives, and capacity-building occurred at a more systemic level. Several patients were often discussed in a single session, and the HM/HC consultant's role often shifted from "expert" to facilitator of team interaction. The support of senior health management in partnering with health regions and First Nations communities was vital to building linkages with primary care physicians, but the natural evolution of rapport between program consultants and physicians was perhaps the more important factor in influencing productive liaisons.

Anecdotally, practitioners perceived that case conceptualization was improved when a child's history and presenting concerns were articulated by a collaboration of care providers. The gathering of this group led to a sense of cohesion and mutual understanding of roles that then facilitated the efficient formulation and implementation of practical and measurable interventions. These interventions were accomplished in partnership with the available local resources. Skill acquisition was enhanced by the opportunity to observe the consultant and/or psychiatrist at work in vivo.

Videoconferencing showed gradual growth as a component of HM/HC's clinical consultation; to date, there have been 36 video-based consults. The reasons for the delayed growth in this area could be the lack of familiarity with the equipment, the reluctance of PCPs to travel to the nearest videoconferencing facility, the time commitment required of physicians, and the tendency to remain with more traditional forms of practice. Generally, once primary care physicians tried this mode of communication, their feedback was positive; their concerns focused on technological, rather than clinical, issues.

The collaboration between HM/HC and Treaty 7 First Nations was the first of its kind in Canada to focus on children's mental health. Developing these partnerships required knowledge of key protocols when working with First Nations tribes and an understanding of the intricacies of jurisdictional and historical issues surrounding First Nations communities.

In Alberta, the First Nations communities of Treaty 7 span three health regions and comprise mainly the tribes of the Blackfoot nation who share a similar culture and language, and the Athabascanspeaking tribes located west of the city of Calgary. Each of the tribes is governed by an elected chief and council who are advised by the Treaty 7 Management Corporation; health care for each tribe is administered by directors who are part of this corporation. The process of building alliances began at this level of governance before progressing to the reserve level.

Key stakeholders were identified as (a) the area agency management team (including education, child welfare, social services, and mental health services) and (b) primary care physicians and elders. The HM/HC consultant was instrumental in helping to craft interagency agreements, a necessary step to developing cooperation and support between primary care physicians and other front-line workers. The process of developing a consultative liaison was slow and deliberate and took between 6 months 
and 1 year. To date, family physicians in two Tribal Health Clinics have welcomed the opportunity for consultation, and regularly scheduled clinics are held to discuss the care of individual patients.

The First Nations communities faced many challenges that needed to be recognized and addressed. Consultation meetings were often cancelled due to deaths in these communities; for the most part there was not enough time to grieve before the next wave of losses hit. These losses take an exhausting toll on front-line workers and primary care physicians, often resulting in restricted service resources.

The pace in rural First Nations communities was slower than in urban centres, and access to communications technology was limited: Most Internet connections have not yet been upgraded to high speed, which restricted professionals' access to online education. Faxing was the preferred method of communicating between agencies; email was less common. Despite the geographic dispersion of the communities of Treaty 7, video consultation was not utilized widely. Most front-line providers appeared uncomfortable with unfamiliar technology as a resource tool. Reserve social workers reported feeling overwhelmed by such advanced technology. As all of the First Nations clinics were equipped with telehealth technology, training and in-service on the operation of telehealth will be the next step in consultation for First Nations communities.

Despite these challenges, major progress has been realized by $\mathrm{HM} / \mathrm{HC}$ in the last 2 years in its engagement with First Nations communities. This growth in capacity is reflected in the significant increases in clinical consultation activity. There are also more continuing education courses being offered to Aboriginal communities and professionals province-wide with a specific focus on First Nations issues.

\section{CONCLUSION}

Healthy Minds/Healthy Children Outreach Service was developed in 2003 to facilitate transfer of knowledge and skill in the area of child and youth mental health, bring mental health services closer to children and families, and help coordinate services across southern Alberta. The program has encountered some challenges, the major ones being the shift in practice culture for medical practitioners toward collaborative care, insufficient Internet connection in rural areas, and the lack of familiarity of First Nations care providers with some forms of technology. Despite these issues, the program has been successful. Numerous clinical consultations have taken place, including consultations about significant numbers of First Nations children. Resource materials have been disseminated broadly and have been well received. Online continuing professional development has proliferated rapidly and has proven to be highly satisfactory to front-line clinicians. There are indications that the interprofessional dialogue that has occurred is promoting a growing community of practice that will positively affect patient care.

The benefits of these forms of capacity-building are seen as significant. Practitioners, regardless of location, can have access to education and practical tools that are convenient, current, and credible. The resulting interprofessional interaction can reduce the isolation faced by many rurally based clinicians and practitioners serving First Nations communities and alleviate the stressful demands of primary care. At the same time, this collaborative approach offers the public the opportunity to continue to receive care from professionals they already know and trust. 


\section{CHILDREN'S MENTAL HEALTH IN PRIMARY HEALTH CARE}

Reports from practitioners indicate their satisfaction with this service, and feedback from other children's mental health professionals suggests that this program is a well-received innovation. Current program efforts are turning to the design of more empirical measurements of practice change in practitioners as a result of online Continuing Professional Development beyond the traditional measures of participant satisfaction and self-reported indicators. There is also an ongoing need to more objectively evaluate the impact of this service on patient care, which should be the ultimate criterion of success for any clinical/educational program. Patient care will be the focus of HM/HC's evaluation efforts and will guide its development in the years to come.

\section{NOTE}

1. http://hmhc.ca

\section{RÉSUMÉ}

Le programme albertain Healthy Minds/Healthy Children s'insère dans la stratégie de cette province visant une capacité accrue des prestataires de soins primaires d'identifier et de traiter les jeunes ayant des problèmes de santé mentale. Cet article présente l'élaboration et la mise en œuvre de ce programme, et il analyse les résultats préliminaires obtenus. Il note également quelques difficultés auxquelles le programme fait face: la communication avec les collectivités autochtones, le développement des ressources, la prestation de consultations cliniques et de perfectionnement professionnel par Internet. Les données recueillies suggèrent que cette forme de service interprofessionnel, axé sur la collaboration et sur l'éducation, pourrait aider à relever les défis que pose l'accès des jeunes aux soins de santé mentale.

\section{REFERENCES}

Adlaf, E.M., Paglia, A., \& Beitchman, J.H. (2002). The mental health and well-being of Ontario students 19912001: Findings from the OSDUS. Toronto, ON: Centre for Addiction and Mental Health.

Ayala, J.S. (2007). The Distance MSW in Leadership in Human Services Program: A case study. Calgary, AB: University of Calgary.

Barnes, B. (2004). Minding the gap: Can continuing medical education bridge the quality chasm? [Editorial comment]. Journal of the American College of Cardiology, 44, 199-200. Retrieved November 1, 2007, from http://content.onlinejacc.org/cgi/content/full/44/1/199

Bassoppo-Moyo, T.C. (2006). Evaluating eLearning: A front-end, process and post hoc approach. International Journal of Instructional Media, 33, 7-22.

Billings, D.M., \& Rowles, C.J. (2001). Development of continuing nursing education offerings for the World Wide Web. Journal of Continuing Education in Nursing, 32, 107-113.

Canadian Collaborative Mental Health Initiative. (2006). Establishing collaborative initiatives between mental health and primary care services for children and adolescents. A companion to the CCMHI planning and implementation toolkit for health care providers and planners. Mississauga, ON: Author. Retrieved November 19, 2007, from http://ccmhi.ca/en/products/toolkits/documents/EN_CompanionToolkitforChildrenandAdolescents.pdf

Canadian Medical Protective Association. (2005). Collaborative care: A medical liability perspective. Ottawa, ON: Author. Retrieved May 14, 2008, from http://toolkit.cfpc.ca/en/interdisciplinary-collaboration/documents/06_collaborative_care-e1.pdf

Chyung, S.Y. (2001). Systematic and systemic approaches to reducing attrition rates in online higher education. American Journal of Distance Education, 15(3), 36-49. 
Cockburn, K., \& Bernard, P. (2004). Child and adolescent mental health within primary care: A study of general practitioners' perceptions. Child and Adolescent Mental Health, 9, 21-24.

Collaborative Working Group on Shared Mental Health Care. (2000). Shared mental health care in Canada: Current status, commentary and recommendations (Report from the Collaborative Working Group on Shared Mental Health Care). Ottawa, ON: Canadian Psychiatric Association and College of Family Physicians of Canada. Retrieved September 15, 2008, from http://www.cfpc.ca/local/files/Mental/sharedmentalcare.pdf

Craven, M.A., \& Bland, R. (2002). Shared mental health care: A bibliography and overview. Canadian Journal of Psychiatry, 47(2 Suppl. 1), iS-viiiS, 1S-103S.

Curran, V.R., \& Fleet, L. (2005). A review of evaluation outcomes of web-based continuing medical education. Medical Education, 39, 561-567.

Davis, D., O’Brien, M.A., Freemantle, N., Wolf, F.M., Mazmanian, P., \& Taylor-Vaisey, A. (1999). Impact of formal continuing medical education: Do conferences, workshops, rounds and other traditional continuing education activities change physician behavior or health care outcomes? Journal of the American Medical Association, 282, 867-874.

Dolinski, C. (2005). Southern Alberta Child and Youth Health Network - Evaluation report. Calgary, AB: Calgary Health Region. Retrieved October 21, 2007, from http://www.sacyhn.ca/media/pdf/SACYHN_Evaluation_ Report_2005.pdf

Harvey, L. (2004). Analytic quality glossary. Quality Research International. Retrieved August 21, 2007, from http://www.qualityresearchinternational.com/glossary/cpd.htm

Hasselback, P, Halma, L., Wedel, R., \& Janz, L. (2003). Appendix L: Development and impact of an alternative payment plan: Key points from a physician's perspective. In The Taber Integrated Primary Care ProjectTurning vision into reality. Final report. Retrieved October 23, 2007, from http://www.uleth.ca/man/ taberresearch/Appendices/L.pdf

Health Canada. (2002). A report on mental illnesses in Canada. Ottawa, ON: Author. Retrieved November 1, 2007, from http://www.phac-aspc.gc.ca/publicat/miic-mmac/pdf/men_ill_e.pdf

Johnston, M., Smylie, J.A., \& Prince, P.N. (2004). Shared care: Getting started. CPA Bulletin, 36(2), 18, 23-24. Retrieved September 15, 2008, from http://ww1.cpa-apc.org:8080/Publications/Archives/Bulletin/2004/april/ johnston-SF.pdf

Kaner, E., Steven, A., Cassidy, P., \& Vardy, C. (2003). Implementation of a model for service delivery and organisation in mental healthcare: A qualitative exploration of service provider views. Health \& Social Care in the Community, 11, 519-527.

Kataoka, S.H., Zhang, L., \& Wells, K.B. (2002). Unmet need for mental health care among U.S. children: Variation by ethnicity and insurance status. American Journal of Psychiatry, 159, 1548-1555.

Kirby, M.J.L., \& Keon W.J. (2006). Out of the shadows at last: Transforming mental health, mental illness and addiction services in Canada. Ottawa: Standing Senate Committee on Social Affairs, Science and Technology. Retrieved August 18, 2008, from http://www.parl.gc.ca/39/1/parlbus/commbus/senate/com-e/soci-e/ rep-e/rep02may06-e.htm

Kushnir, T., Cohen, A.H., \& Kitai, E. (2000). Continuing medical education and primary physicians' job stress, burnout and dissatisfaction. Medical Education, 34, 430-436.

Kutcher, S. (2007). Mentally ill youth: Meeting service needs. Canadian Medical Association Journal, 176, $417,419$.

Mamary, E.M., \& Charles, P. (2000). On-site to on-line: Barriers to the use of computers for continuing education. Journal of Continuing Education in the Health Professions, 20, 171-175.

McElroy, R. (2004). Canada's shortage of physicians [Letter]. Canadian Family Physician, 50, 349. Retrieved October 31, 2007, from http://www.cfpc.ca/cfp/2004/Mar/vol50-mar-letters-1.asp

McEwan, K., Waddell, C., \& Barker, J. (2007). Bringing children's mental health "out of the shadows." Canadian Medical Association Journal, 176, 471-472.

Morgan, C.K., \& Tam, M. (1999). Unravelling the complexities of distance education student attrition. Distance Education, 20, 96-108. 


\section{CHILDREN'S MENTAL HEALTH IN PRIMARY HEALTH CARE}

Pauzé, E., Gagné, M.A., \& Pautler, K. (2005). Collaborative mental health care in primary health care: A review of Canadian initiatives. Vol. I: Analysis of initiatives. Mississauga, ON: Canadian Collaborative Mental Health Initiative. Retrieved August 15, 2008, from http:/ccmhi.ca/en/products/documents/ 05a_CanadianReviewI-EN.pdf

Peterson, M.W. (1999). Continuing medical education on the Internet: State of the art. Journal of Continuing Education in the Health Professions, 19, 242-249.

Premier's Advisory Council on Health for Alberta. (2001). A framework for reform: A report of the Premier's Advisory Council on Health. Edmonton, AB: Author. Retrieved August 15, 2008, from http://dsp-psd.tpsgc.gc.ca/ Collection-R/LoPBdP/BP/prb0133-e.htm

Richardson, M.L., \& Norris, T.E. (1997). On-line delivery of continuing medical education over the WorldWide Web: An on-line needs assessment. American Journal of Roentgenology, 168, 1161-1164.

Ryan, D., \& Waterston, R. (2000). Transforming continuing education materials for on-line learning. Journal of Telemedicine and Telecare, 6(Suppl. 2), S64-S66.

Saba, F.E. (2000). Student attrition: How to keep your online learner focused. Distance Education Report, 4(14), 1-2.

Steele, M., Stretch, N., Andreychuk, R., Sylvester, H., Rourke, J., Dickie, G., et al. (2004). Teaching children's mental health to family physicians in rural and underserviced areas. CPA Bulletin, 36(5), 8-9.

Steele, M.M., Fisman, S., Dickie, G., Stretch, N., Rourke, J., \& Grindrod, A. (2003). Assessing the need for and interest in a scholarship program in children's mental health for rural family physicians. Canadian Journal of Rural Medicine, 8, 163-170.

Steinhauer, P. (1999). Review of the organization and delivery of children's mental health services in Edmonton and Calgary. Edmonton, AB: Alberta Mental Health Board.

Towle, A. (2000). Shifting the culture of continuing medical education: What needs to happen and why is it so difficult? Journal of Continuing Education in the Health Professions, 20, 208-218. 